\title{
Molecular biology of malignant mesothelioma
}

\author{
Yoshitaka Sekido
}

Received: 27 June 2007 / Accepted: 15 September 2007/Published online: 28 February 2008

(C) The Japanese Society for Hygiene 2008

\begin{abstract}
Human malignancies develop via a multi-step that involves the accumulation of several key gene alterations with associated genetic and epigenetic events. Although malignant mesothelioma (MM) has been demonstrated to be clearly correlated with asbestos exposure, it remains poorly understood how asbestos fibers confer key gene alterations and induce cellular transformation in normal mesothelial cells, which results in the acquisition of malignant phenotypes, including deregulated cell proliferation and invasion. Malignant mesothelioma presents with the frequent inactivation of tumor suppressor genes of $p 16^{I N K 4 a} / p 14^{A R F}$ on chromosome 9p21 and neurofibromatosis type 2 (NF2) on chromosome 22q12, with the latter being responsible for the NF2 familial cancer syndrome. In contrast, MM shows infrequent mutation of the p53 gene, which is one of the most frequently mutated tumor suppressor genes in human malignancies. Genetic abnormalities of oncogenes have also been studied in MM, but no frequent mutations have been identified, including the epidermal growth factor receptor $(E G F R)$ and $K-R A S$ genes. Recent studies have suggested the activation of other receptor tyrosine kinases, including Met, and the deregulations of mitogen-activated protein kinase (MAPK) and phosphatidylinositol-3-kinase (PI3K)-AKT signaling cascades, although the alterations responsible for their activation are still not clear. Thus, further genome-wide
\end{abstract}

This article is based upon the Symposium Lecture Series, "To Know Malignant Mesothelioma," at the 77th Annual Meeting of the Japanese Society for Hygiene held in Osaka, Japan on March 25-28, 2007.

Y. Sekido $(\bowtie)$

Division of Molecular Oncology, Aichi Cancer Center Research Institute, 1-1 Kanokoden, Chikusa-ku, Nagoya 464-8681, Japan

e-mail: ysekido@aichi-cc.jp studies of genetic and epigenetic alterations as well as detailed analyses of deregulated signaling cascades in MM are necessary to determine the molecular mechanisms of MM, which would also provide some clues for establishing a new molecular target therapy for MM.

Keywords Asbestos - Malignant mesothelioma . Molecular target therapy - Oncogene .

Tumor suppressor gene

\section{Introduction}

Malignant mesothelioma (MM), a highly lethal neoplasm arising primarily in the pleural, peritoneal, or pericardial cavity, is thought to develop from surface mesothelial cells [1]. In up to $80 \%$ of patients, MM occurs within approximately 30-40 years following exposure to asbestos [2]. Since patients with MM are usually diagnosed at advanced stages and MM is refractory to conventional therapy, the prognosis of patients with $\mathrm{MM}$ is very poor. The median survival of patients with malignant pleural mesothelioma (MPM) is 7-11 months after diagnosis, despite the recent advancements in chemotherapeutical modalities combining cisplatin and antifolates such as pemetrexed or raltitrexed $[3,4]$.

The regulation of asbestos occurred relatively late in Japan, with the result that the number of patients with MM is expected to increase year by year, with the peak incidence predicted around 2030-2040 [5]. To date, in Japan approximately 1000 deaths can be attributed to MM. It has been estimated that 250,000 people will die of MPM in Europe during the next three decades and in the United States, 2500-3000 new cases are diagnosed each year [6, 7]. These numbers are attributable to the wide use of 
asbestos in various industrial and building materials, resulting in exposure to asbestos not only among workers in factories and/or at construction and demolition sites but also by ordinary citizens including family members of the workers and local residents near the factories and affected sites.

About $80 \%$ of MM develops in the pleura, $20 \%$ in the peritonea, and less than $1 \%$ in the pericardium. The pathologically, epithelial type accounts for about $60 \%$ of all cases, the sarcomatous type for about $20 \%$, and the biphasic type with both components ranges around $20 \%$. Since MM is a relatively rare human malignancy, both basic and clinical studies have lagged far behind those on other common types of malignancies, such as lung, colon, and breast cancers. However, many researchers are now focusing their efforts on identifying the mechanisms and key genes in MM development with the aim of using this information to develop new diagnostic tools and target molecules of new therapy.

\section{Asbestos-induced oncogenesis}

Up to $80 \%$ of mesothelioma patients have been exposed to asbestos, thus establishing a clear link between asbestos exposure and MM development [1]. Many animal models have also demonstrated the carcinogenicity of asbestos. There are six types of asbestos: amphiboles-amosite (brown asbestos), crocidolite (blue asbestos), anthophyllite, actinolite, and tremolite, the serpentine chrysotile (white asbestos). However, it has not yet been clearly determined whether asbestos fibers act directly on the mesothelial cells or whether they indirectly cause mesothelioma. Several plausible explanations have been put forward on how asbestos fibers can cause MPM [1,8]. One such suggestion is that asbestos fibers mechanically induce pleural irritation: long and thin asbestos fibers can be inhaled deeply into the lungs, penetrating and repeatedly scratching the mesothelial surface, resulting in prolonged cycles of damage, repair, and local inflammation. Alternatively, asbestos fibers can also mechanically interfere with the mitotic process of the cell cycle by disrupting the mitotic spindle, which may result in chromosomal abnormalities and aneuploidy. A third proposal is that highly reactive oxygen species (ROS) and reactive nitrogen species (RNS) are induced by asbestos, leading to DNA damage and strand breaks. A ramification of the interaction of long fibers with cells is frustrated phagocytosis and a prolonged oxidative burst. Finally, asbestos can induce cytokines and growth factors, such as transforming growth factor- $\beta$ (TGF- $\beta$ ) and platelet-derived growth factor (PDGF), as well as transcription factors, such as nuclear factor kappa $\mathrm{B}$ $(\mathrm{NF}-\kappa \mathrm{B})$ and activator protein-1 (AP-1). Additionally, in rat mesothelial cells, crocidolite asbestos was found to cause autophosphorylation of epidermal growth factor receptor (EGFR) [9].

\section{Inactivation of tumor suppressor genes in MPM}

The most frequently inactivated tumor suppressor genes (TSGs) in human malignancies are $p 53$ and $p 16^{I N K 4 a}$ / $p 14^{A R F}$. Most MPMs show frequent inactivation of $p 16^{I N K 4 a} / p 14^{A R F}$, although only $20-25 \%$ of these show a mutation of $p 53$. Analysis of primary samples of MPM revealed that over $70 \%$ of the samples showed downregulation of $p 16^{I N K 4 a} / p 14^{A R F}[10,11]$. Using established MPM cell lines, Taniguchi et al. [12] found that almost all of the cell lines had a homozygous deletion of the $p 16^{I N K 4 a}$ / $p 14^{A R F}$ gene locus. The $p 16^{I N K 4 a}$ gene product controls the cell cycle via the cyclin-dependent kinase 4 (CDK4)/ Cyclin D-RB pathway, while the $p 14^{A R F}$ gene product regulates p53 through inactivation of the human homolog of mouse double minute 2 (HDM2), which is an upstream regulator of $\mathrm{p} 53$. Thus, the homozygous deletion of p16 $6^{I N K 4 a} / p 14^{A R F}$ indicates the inactivation of two major tumor-suppressing pathways of RB and p53 in the cell.

The loss of chromosome 22q12 is frequently detected in MPM. The neurofibromatosis type 2 (NF2) gene, which had been initially identified as a target gene of NF2 familial cancer syndrome, has also been shown to be the target gene of the 22q12 loss in MPM [13, 14]. Neurofibromatosis type 2 patients frequently develop vestibular schwannoma, meningioma, and other neuronal malignancies, while sporadic tumors of these types of cancer also harbor a NF2 mutation [15]. However, to date, there has been no published report of NF2 patients showing a higher susceptibility to MPM. The NF2 gene is inactivated by homozygous deletion, nonsense mutation, or missense mutation in MPMs. Bianchi et al. [14] and Sekido et al. [13] reported that about $40 \%$ of MPMs showed the genetic mutation of NF2; among the $60 \%$ of MPMs without this $N F 2$ genetic mutation, about $20 \%$ showed a down-regulation of NF2. In total, 50-60\% of MPMs showed inactivation of NF2. In an animal model, an established line of $N f 2( \pm)$ knockout mice were shown to develop MPMs in the earlier stage and, more frequently, after asbestos exposure [16]. Remarkably, similar to human $\mathrm{MM}$, tumors from $N f 2( \pm)$ mice showed frequent homozygous deletions of the $p 16^{I n k 4 a} / p 19^{A r f}$ locus and adjacent $p 15^{\text {Ink } 4 b}$ tumor suppressor gene.

The NF2 gene encodes a membrane-cytoskeletonassociated protein, Merlin, an adaptor protein with a FERM (four-point-one, ezrin, radixin, and moesin) domain, which transduces a growth-regulatory signal [17]. Merlin has been shown to interact with several proteins, including 
CD44, ezrin radixin moesin (ERM) proteins, p21-activated kinase 1 (PAK1), and loses its tumor-suppressing activity with phosphorylation at serine 518 . This serine 518 site is phosphorylated with RAC/PAK1 and dephosphorylated with myosin phosphatase targeting subunit 1-protein phosphatase $1 \delta$ (MYPT-1-PP1 $\delta$ ) [18]. The activity of Merlin is thought to be regulated by cell-adhesion (adherence junction), cell-extracellular matrix adhesion, or extracellular growth signals. The downstream signaling of Merlin is mediated by Hippo cascade, which was initially identified via genetic studies in Drosophila [19]. Thus, Merlin is thought to be one of the key molecules in the signaling cascades that determine the properties of invasion, cell growth, and survival of malignant mesothelioma cells (Fig. 1).

\section{Activation of oncogenes in MPM}

Simian virus 40 (SV40) is a double-stranded DNA polyomavirus of monkey origin, which has been suggested to be associated with MM development [20]. Since the polio vaccine that was used between 1954 and 1963 was widely contaminated with SV40, vaccination was proposed as a plausible vector for the widespread SV40 infection of the human population. However, even people who had not received a polio vaccination were found to be infected with SV40, leaving unanswered the questions as to how SV40 virus infected humans.

Since SV40 expresses large T and small T antigens, and the large $\mathrm{T}$ antigen binds and inactivates the p53 and RB tumor suppressors, SV40 infection has been recognized as one of primary mechanisms of mesothelioma development pathogenetically. Intrapleural infection of SV40 in hamsters induces mesothelioma development by 6 months after administration, and SV40 induces Met, Notch-1, and telomerase activity, which also supports the functional roles of SV40.

However, most of the studies showing a strong relationship between SV40 and MM consisted exclusively of PCR-based assays in which the simple amplification of specific segments was considered to indicate a positive result; otherwise, the data were conflicting and reproducibility was limited. The results from several more recent studies suggest that such results were false-positives due to contamination by plasmids that were in general use in many of the laboratories common among the studies [21]. Consequently, the involvement of SV40 in malignant mesothelioma remains controversial.

The activation of receptor tyrosine kinase (RTK) family members has been investigated in MM. Among these, the Met oncogene has been shown to be frequently expressed

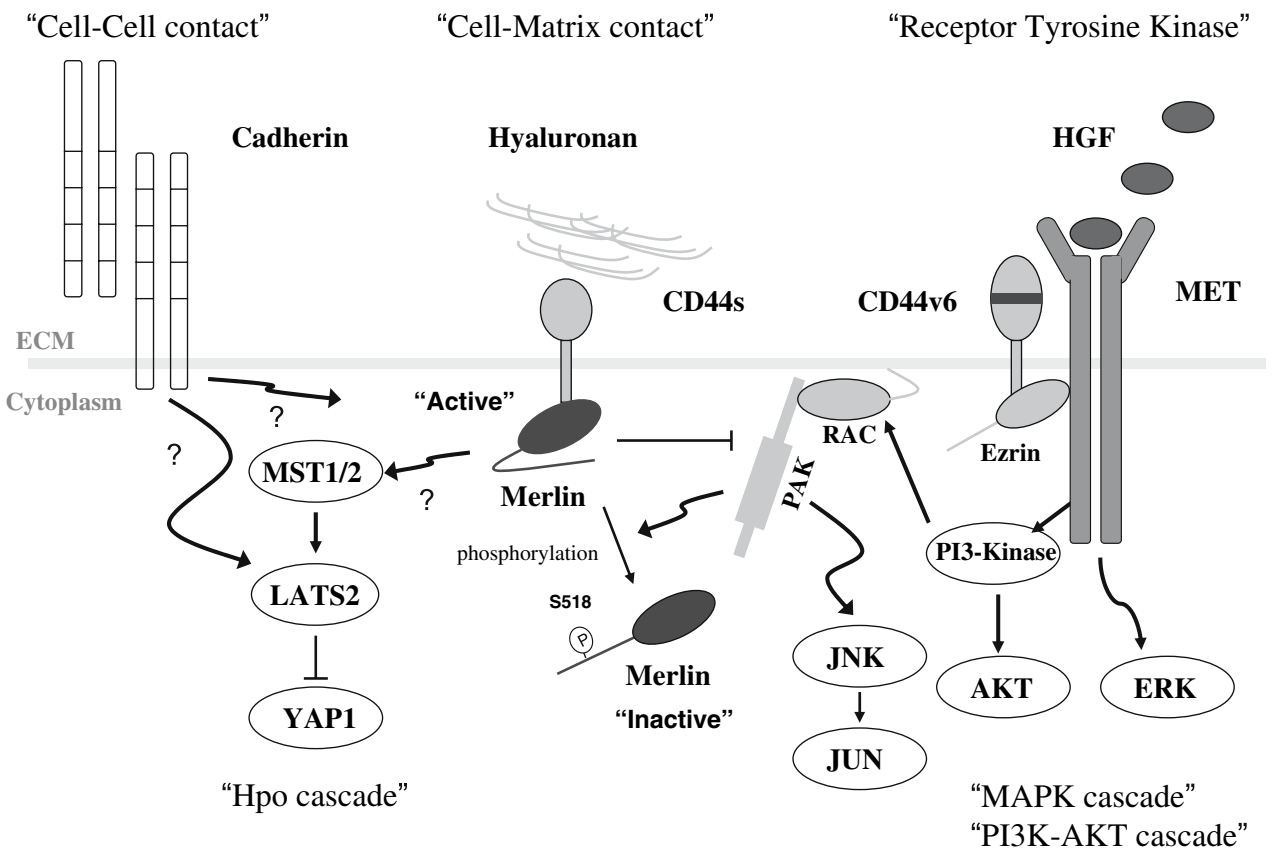

Fig. 1 A schematic representation of the neurofibromatosis type 2 (NF2) gene product, Merlin, and its possible related signaling cascades. Signals from extracellular environments are transduced via cell-cell contact, cell-matrix contact, or growth factors and then transmitted to signaling cascades, such as the MAPK (mitogenactivated protein kinase), PI3K (PI3 phosphatidylinositol-3 kinase)-
AKT, and Hpo (Hippo) cascades, which are regulated by Merlin and its homologues [ezrin radixin moesin (ERM) proteins], such as Ezrin. These signaling cascades are often deregulated in malignant mesothelioma (MM) cells. ECM Extracellular matrix, ERK extracellular signal-regulated kinase $J N K$ JUN N-terminal kinase, $M A P K$ mitogenactivated protein, PI3 phospatidylinositol-3 
in primary tumors and cell lines of MM [22]. Hepatocyte growth factor (HGF) is a ligand of Met, and HGF-MET plays a role in the mitogen-activated protein kinase (MAPK) and phosphatidylinositol-3-kinase (PI3K)-AKT cascades. The PI3K-AKT cascade has also been shown to be activated in MM. Furthermore, MMs with positive AKT phosphorylation status show phosphorylated (activated) mTOR (mammalian target of rapamycin), which is one of the downstream molecules of AKT. Thus, the activation of the PI3K-AKT-mTOR signaling cascade in MM is thought to be induced by activation of the upstream HGF-MET (Fig. 1). Additionally, although EGFR, another RTK which is frequently mutated in Asian female adenocarcinoma of the lung, was shown to be overexpressed in $56 \%$ of primary tumors, no EGFR mutation was documented [23].

Malignant mesothelioma cell lines produce many other growth factors and cytokines [1]. Platelet-derived growth factor, TGF- $\beta$, insulin-like growth factor (IGF) have been studied in depth. In addition, factors involving angiogenic pathways have also been demonstrated to be expressed in MM cells, including interleukin (IL)-6, IL-8, fibroblast growth factors (FGFs), and vascular endothelial growth factors (VEGFs) [24].

\section{Searching for new key genes in malignant mesothelioma}

Since only a small number of oncogenes or TSGs with genetic alterations has been identified in MM, other, as yet unidentified genes may well be responsible for its development. Traditional allelotyping and karyotype analyses have revealed nonrandom chromosomal abnormalities, including 1p, 3p, 4p15.1-p15.3, 4q25-q26, 4q33-q34, 6q, 9p, 14q11.1-q12, 14q23-q24, and 22q [25, 26]. A comparative genomic hybridization $(\mathrm{CGH})$ technique has recently been introduced to search for additional genes that are potentially involved in MM biology. New alteration regions have been identified, including 1q, 4q, 5p, 6p, 7p, 8p, 8q, 10p13-pter, 13q, 14q, 15q, 17p12-pter, 17q, and 20, in which new cancer-associated genes of MM may be harbored [27, 28]. A recent study of array-based CGH analysis with MMs from a total of 22 individuals identified high-copy gain at $1 \mathrm{p} 32$, which includes the $J U N$ protooncogene [12]. JUN is a transcription factor and functions as homo- or hetero-dimerization with FOS to form the transcription factor AP-1, which can bind to the promoter region of intermediate genes involved in cell division and other cell functions. Both crocidolite and chrysotile asbestos reportedly caused increases in the expression of $J U N$ and FOS in rat pleural mesothelial cells [29]. Since the gene amplification of $J U N$ was identified in a subset of MPM tumors, it was suggested that there were some strong and persistent factors for JUN activation during the development of MPM tumor cells. A more recent study reported an activated mutation of $N-R A S$ in three of 38 MMs [30].

Finally, expression profiling using microarray has been also studied to identify specific gene expression changes in MM compared with normal mesothelium [31-34]. Several new candidate oncogenes and TSGs of MM were proposed, and patient prognosis was shown to be predictable with the differences of gene expression profiling.

\section{Application for molecular target therapy}

Malignant mesothelioma is a highly aggressive tumor, and the patient prognosis with advanced-stage MM is very poor. Combination chemotherapy with cisplatin and antifolate has recently been shown to be superior to cisplatin alone [3, 4]. Although several molecular target therapies have been tested, no satisfactory results have been obtained to date. For example, a phase II study of an EGFR inhibitor, gefitinib, was conducted for 43 patients with previously untreated MM [35]. Although 97\% of patients with MM had EGFR overexpression, gefitinib was not active in MM and EGFR expression did not correlate with failure-free survival. Imatinib, another tyrosine kinase inhibitor known to affect both Kit and PDGF $\alpha$ (and $\beta$ ) receptors, has also been shown to have limited efficacy for MPM [36].

A recent microarray analysis on 99 MPM detected advanced-stage, sarcomatous histology and the $p 16^{I N K 4 a /}$ $p 14^{A R F}$ homozygous deletion to be significant adverse prognostic factors [37]. The same study also found that more aggressive MPM expressed higher levels of Aurora kinases $\mathrm{A}$ and $\mathrm{B}$, which are serine/threonine kinases with multiple roles in mitotic progression. Thus, the role of Aurora kinases is of interest due to the recent development of their small-molecule inhibitors. In addition, a smallmolecule inhibitor of TGF $\beta$ type I receptor has been shown to inhibit murine mesothelioma tumor growth in vivo [38].

\section{Summary}

The long latency period between asbestos exposure and tumor development implies that multiple-and likely diverse-genetic changes are required for the malignant transformation of mesothelial cells. Many studies have been conducted to determine the underlying key genetic and epigenetic events responsible for the development of MPM, some of which may be directly caused by asbestos fibers. New animal models of MM and human MM cell lines are also being established to present more useful tools for detailed analyses of the carcinogenesis of MM and the 
development of new therapeutic modalities [16, 39]. Thus, more in-depth knowledge of key gene alteration, specific expression profiling, and other fundamental abnormalities at the cellular, intercellular, and tissue levels in MM cells will be of great help in developing future strategies for potential molecular targets as well as other therapeutic modalities, such as immunotherapy.

Acknowledgments This work was supported by a Special Coordination Fund for Promoting Science and Technology from the Ministry of Education, Culture, Sports, Science and Technology (H18-1-3-31). I thank Dr. Hideki Murakami, Dr. Yutaka Kondo, Dr. Hirotaka Osada, and Dr. Tetsuo Taniguchi for their helpful comments. I regret the lack of citations for many important observations in the text, but their omission is made necessary by restrictions on the preparation of review manuscripts.

\section{References}

1. Pass HI, Vogelzang N, Hahn S, Carbone M. Malignant pleural mesothelioma. Curr Probl Cancer. 2004;28:93-174.

2. Carbone M, Kratzke RA, Testa JR. The pathogenesis of mesothelioma. Semin Oncol. 2002;29:2-17.

3. Vogelzang NJ, Rusthoven JJ, Symanowski J, Denham C, Kaukel E, Ruffie P, et al. Phase III study of pemetrexed in combination with cisplatin versus cisplatin alone in patients with malignant pleural mesothelioma. J Clin Oncol. 2003;21:2636-44.

4. van Meerbeeck JP, Gaafar R, Manegold C, Van Klaveren RJ, Van Marck EA, Vincent M, et al. Randomized phase III study of cisplatin with or without raltitrexed in patients with malignant pleural mesothelioma: an intergroup study of the European Organisation for Research and Treatment of Cancer Lung Cancer Group and the National Cancer Institute of Canada. J Clin Oncol. 2005;23:6881-9.

5. Murayama T, Takahashi K, Natori Y, Kurumatani N. Estimation of future mortality from pleural malignant mesothelioma in Japan based on an age-cohort model. Am J Ind Med. 2006;49:1-7.

6. Peto J, Decarli A, La Vecchia C, Levi F, Negri E. The European mesothelioma epidemic. Br J Cancer. 1999;79:666-72.

7. Robinson BW, Lake RA. Advances in malignant mesothelioma. N Engl J Med. 2005;353:1591-1603.

8. Robinson BW, Musk AW, Lake RA. Malignant mesothelioma. Lancet. 2005;366:397-408.

9. Faux SP, Houghton CE, Hubbard A, Patrick G. Increased expression of epidermal growth factor receptor in rat pleural mesothelial cells correlates with carcinogenicity of mineral fibres. Carcinogenesis. 2000;21:2275-80.

10. Kratzke RA, Otterson GA, Lincoln CE, Ewing S, Oie H, Geradts $\mathrm{J}$, et al. Immunohistochemical analysis of the p16INK4 cyclindependent kinase inhibitor in malignant mesothelioma. J Natl Cancer Inst. 1995;87:1870-5.

11. Cheng JQ, Jhanwar SC, Klein WM, Bell DW, Lee WC, Altomare DA, et al. p16 alterations and deletion mapping of 9p21-p22 in malignant mesothelioma. Cancer Res 1994;54:5547-51.

12. Taniguchi T, Karnan S, Fukui T, Yokoyama T, Tagawa H, Yokoi $\mathrm{K}$, et al. Genomic profiling of malignant pleural mesothelioma with array-based comparative genomic hybridization shows frequent non-random chromosomal alteration regions including JUN amplification on 1p32. Cancer Sci. 2007;98:438-46.

13. Sekido Y, Pass HI, Bader S, Mew DJ, Christman MF, Gazdar AF, et al. Neurofibromatosis type 2 (NF2) gene is somatically mutated in mesothelioma but not in lung cancer. Cancer Res. 1995;55:1227-31.

14. Bianchi AB, Mitsunaga SI, Cheng JQ, Klein WM, Jhanwar SC, Seizinger B, et al. High frequency of inactivating mutations in the neurofibromatosis type 2 gene (NF2) in primary malignant mesotheliomas. Proc Natl Acad Sci USA. 1995;92:10854-8.

15. Baser ME. The distribution of constitutional and somatic mutations in the neurofibromatosis 2 gene. Hum Mutat. 2006;27:297306.

16. Altomare DA, Vaslet CA, Skele KL, De Rienzo A, Devarajan K, Jhanwar SC, et al. A mouse model recapitulating molecular features of human mesothelioma. Cancer Res. 2005;65:8090-5.

17. McClatchey AI, Giovannini M. Membrane organization, tumorigenesis-the NF2 tumor suppressor, Merlin. Genes Dev. 2005;19: 2265-77.

18. Jin H, Sperka T, Herrlich P, Morrison H. Tumorigenic transformation by CPI-17 through inhibition of a merlin phosphatase. Nature. 2006;442:576-9.

19. Hamaratoglu F, Willecke M, Kango-Singh M, Nolo R, Hyun E, Tao C, et al. The tumour-suppressor genes NF2/Merlin and expanded act through Hippo signaling to regulate cell proliferation and apoptosis. Nat Cell Biol. 2006;8:27-36.

20. Gazdar AF, Butel JS, Carbone M. SV40, human tumours: myth, association or causality? Nat Rev Cancer. 2002;2:957-64.

21. Lopez-Rios F, Illei PB, Rusch V, Ladanyi M. Evidence against a role for SV40 infection in human mesotheliomas, high risk of false-positive PCR results owing to presence of SV40 sequences in common laboratory plasmids. Lancet. 2004;364:1157-66.

22. Jagadeeswaran R, Ma PC, Seiwert TY, Jagadeeswaran S, Zumba O, Nallasura V, et al. Functional analysis of c-Met/hepatocyte growth factor pathway in malignant pleural mesothelioma. Cancer Res. 2006;66:352-61.

23. Destro A, Ceresoli GL, Falleni M, Zucali PA, Morenghi E, Bianchi $\mathrm{P}$, et al. EGFR overexpression in malignant pleural mesothelioma. An immunohistochemical and molecular study with clinico-pathological correlations. Lung Cancer. 2006;51: 207-15.

24. Galffy G, Mohammed KA, Dowling PA, Nasreen N, Ward MJ, Antony VB. Interleukin 8: an autocrine growth factor for malignant mesothelioma. Cancer Res. 1999;59:367-71.

25. Murthy SS, Testa JR. Asbestos, chromosomal deletions, tumor suppressor gene alterations in human malignant mesothelioma. J Cell Physiol. 1999;180:150-7.

26. Taguchi T, Jhanwar SC, Siegfried JM, Keller SM, Testa JR. Recurrent deletions of specific chromosomal sites in $1 \mathrm{p}, 3 \mathrm{p}$, 6q, 9p in human malignant mesothelioma. Cancer Res. 1993;53: 4349-55.

27. Balsara BR, Bell DW, Sonoda G, De Rienzo A, du Manoir S, Jhanwar SC, et al. Comparative genomic hybridization and loss of heterozygosity analyses identify a common region of deletion at 15q11.1-15 in human malignant mesothelioma. Cancer Res. 1999;59:450-4.

28. Krismann M, Muller KM, Jaworska M, Johnen G. Molecular cytogenetic differences between histological subtypes of malignant mesotheliomas: DNA cytometry, comparative genomic hybridization of 90 cases. J Pathol. 2002;197:363-71.

29. Heintz NH, Janssen YM, Mossman BT. Persistent induction of c-fos, c-jun expression by asbestos. Proc Natl Acad Sci USA. 1993;90:3299-303.

30. Thomas RK, Baker AC, Debiasi RM, Winckler W, Laframboise $\mathrm{T}$, Lin WM, et al. High-throughput oncogene mutation profiling in human cancer. Nat Genet. 2007;39:347-51.

31. Singhal S, Wiewrodt R, Malden LD, Amin KM, Matzie K, Friedberg J, et al. Gene expression profiling of malignant mesothelioma. Clin Cancer Res. 2003;9:3080-97. 
32. Gordon GJ, Rockwell GN, Jensen RV, Rheinwald JG, Glickman JN, Aronson JP, et al. Identification of novel candidate oncogenes and tumor suppressors in malignant pleural mesothelioma using large-scale transcriptional profiling. Am J Pathol. 2005;166:1827-40.

33. Hoang CD, D'Cunha J, Kratzke MG, Casmey CE, Frizelle SP, Maddaus MA, et al. Gene expression profiling identifies matriptase overexpression in malignant mesothelioma. Chest. 2004; 125:1843-52.

34. Gordon GJ, Jensen RV, Hsiao LL, Gullans SR, Blumenstock JE, Richards WG, et al. Using gene expression ratios to predict outcome among patients with mesothelioma. J Natl Cancer Inst. 2003;95:598-605.

35. Govindan R, Kratzke RA, Herndon JE, 2nd, Niehans GA, Vollmer R, Watson $\mathrm{D}$, et al. Gefitinib in patients with malignant mesothelioma: a phase II study by the Cancer and Leukemia Group B. Clin Cancer Res. 2005;11:2300-4.
36. Mathy A, Baas P, Dalesio O, van Zandwijk N. Limited efficacy of imatinib mesylate in malignant mesothelioma: a phase II trial. Lung Cancer. 2005;50:83-6.

37. Lopez-Rios F, Chuai S, Flores R, Shimizu S, Ohno T, Wakahara $\mathrm{K}$, et al. Global gene expression profiling of pleural mesotheliomas: overexpression of aurora kinases and P16/CDKN2A deletion as prognostic factors and critical evaluation of microarray-based prognostic prediction. Cancer Res. 2006;66:2970-9.

38. Suzuki E, Kim S, Cheung HK, Corbley MJ, Zhang X, Sun L, et al. A novel small-molecule inhibitor of transforming growth factor beta type I receptor kinase (SM16) inhibits murine mesothelioma tumor growth in vivo and prevents tumor recurrence after surgical resection. Cancer Res. 2007;67:2351-9.

39. Usami N, Fukui T, Kondo M, Taniguchi T, Yokoyama T, Mori S, et al. Establishment and characterization of four malignant pleural mesothelioma cell lines from Japanese patients. Cancer Sci. 2006;97:387-94. 